\title{
Ileal Duplication Cyst: A Case Report
}

\author{
Dr. Sreelekshmi S. $\mathrm{N}^{1^{*}}$, Dr. Lillykutty Pothen ${ }^{2}$, Dr. Sankar S ${ }^{3}$
}

${ }^{1}$ Junior Resident Pathology, Govt. Medical College Kottayam, Kerala, India

${ }^{2}$ Additional Professor Pathology, Govt. Medical College Kottayam, Kerala, India

${ }^{3}$ Professor and HOD Pathology, Govt. Medical College Kottayam, Kerala, India

DOI: $\underline{10.36348 / \text { sjpm.2021.v06i03.006 }}$

| Received: 02.03.2021 | Accepted: 19.03.2021 | Published: 25.03.2021

*Corresponding author: Dr. Sreelekshmi S. N

\section{Abstract}

Gastrointestinal duplications are rare congenital malformations that may vary greatly in presentation, size, location and symptoms. They can present as solid/cystic tumors, intussusception, perforation or bleeding. A high index of suspicion is needed in all such cases. Radiologic studies may not be enough for a diagnosis. We present a case of ileal duplication cyst in a 2 month old baby who presented with features of abdominal obstruction. Suspecting it to be an infected mesenteric cyst from radiological studies, surgical excision with anastomosis of the ileal segment was done. The histopathological examination of the resected specimen diagnosed it as an enteric duplication cyst.

Keywords: Ileal duplication cyst, tubular, cystic, mesenteric.

Copyright ( $\odot 2021$ The Author(s): This is an open-access article distributed under the terms of the Creative Commons Attribution 4.0 International License (CC BY-NC 4.0) which permits unrestricted use, distribution, and reproduction in any medium for non-commercial use provided the original author and source are credited.

\section{INTRODUCTION}

Ileal duplication cysts are rare congenital anomalies characterized by an abnormal portion of intestine attached to or intrinsic with the normal bowel [1]. It was first reported in 1733 by Calder [2]. It has an incidence of 1 in 4500 births [3]. $85 \%$ of the duplications present before the age of two [4]. It can be either symptomatic/ may present with vague abdominal symptoms or rarely as acute abdomen. They are often mistaken for acute appendicitis/mesenteric cyst. This is the case report of an ileal duplication cyst in a 2 month old baby which was mistaken for acute appendicitis.

\section{CASE REPORT}

A 2 month old male baby presented to the pediatric surgery department with sudden onset multiple episodes of vomiting and incessant cry. On examination a swelling was palpable in the right lower quadrant of abdomen which was firm and mobile. Ultrasonography of the abdomen revealed a large right iliac fossa cyst with internal echoes possibly mesenteric cyst. CECT abdomen also highlighted presence of a thin walled cyst in the lower abdomen involving the mesentry measuring $6.6 \times 5.3 \times 5.2 \mathrm{~cm}$ raising the possibility of a mesenteric cyst with suspicion of hemorrhage or infection. The child was operated and intra operatively there was a cystic lesion in the mesentry $30 \mathrm{~cm}$ from the ileo ceacal junction. No intussusception was seen. Hence a segment of ileum with attached mass was removed and ends anastomosed.

\section{GROSS SPECIMEN}

We received the segment of ileum with attached cyst in mesentry. The ileal segment measuring $2.5 \mathrm{~cm}$ and intact cystic mass measuring $4.3 \times 4 \times 3$. The surface of the cyst was smooth. Cutting opening, the mass revealed a uniloculated cyst with shaggy inner wall. The lumen contained dark brown material. The ileal mucosa was unremarkable and there was no connection with the cyst. There was no perforation or gangrenous change.

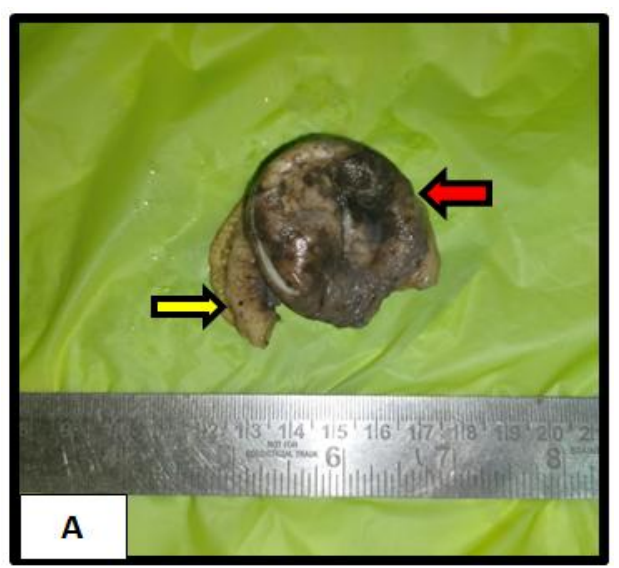

A) Image of resected segment of ileum (yellow arrow) with attached cystic mass (red arrow) in the mesentry 


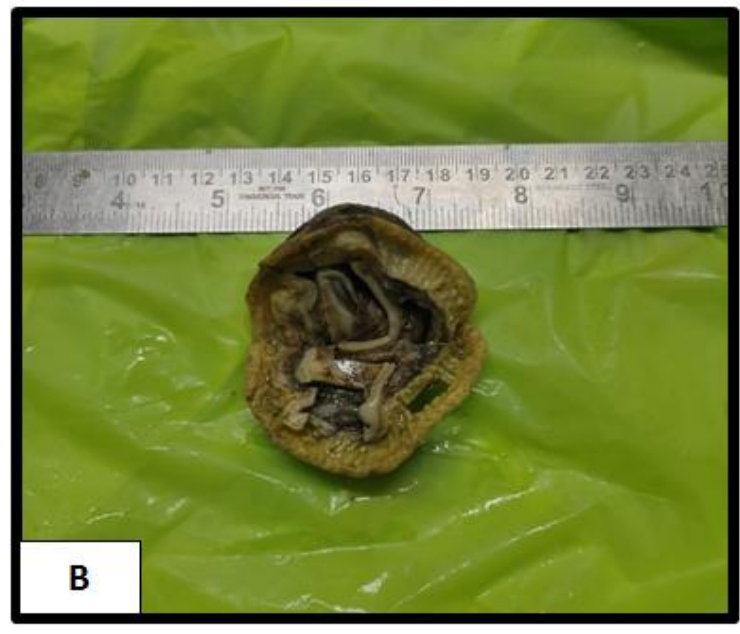

B) Cut section of cystic mass

Microscopy of the cyst wall showed all layers of bowel - mucosa, sub mucosa, muscularis, the muscular layer is common as that of adjacent ileum. The adjacent ileal wall was normal and both resected ends of the ileum were viable.

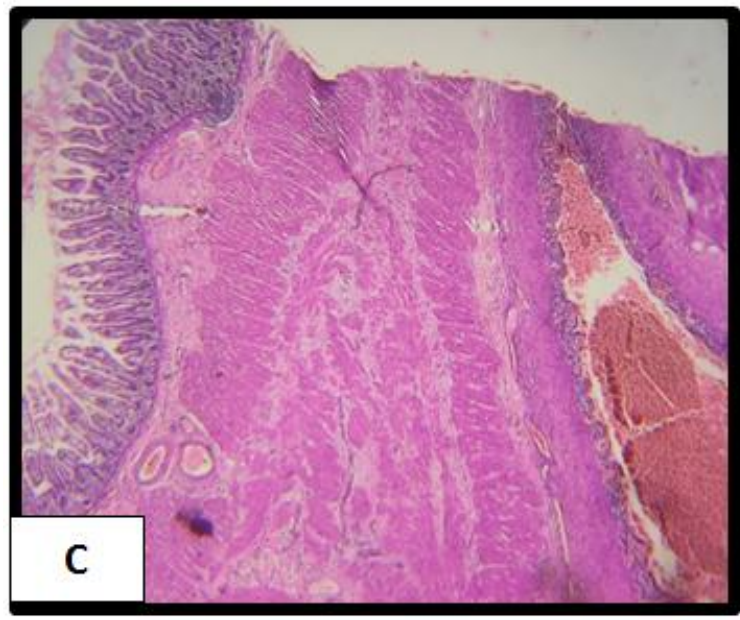

C) Low power view showing ileal mucosa (left) and the mucosa of duplication cyst (right) with the common muscular wall

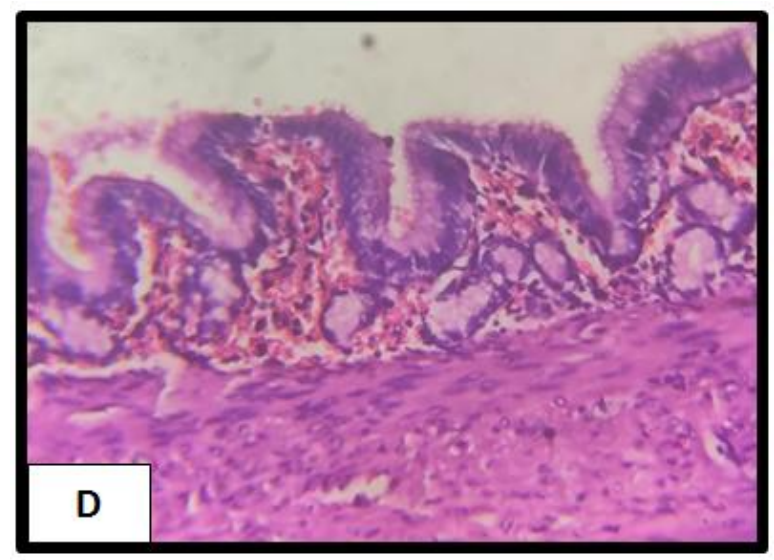

D) High power view of the mucosa of duplication cyst

\section{DISCUSSION}

Gastrointestinal duplication cysts are uncommon congenital anomalies, $80 \%$ of which are diagnosed before 2 years of age [4]. They are of two types in general, cystic which accounts for $80 \%$ of the cases that are spherical in shape and not communicating with the bowel lumen. Remaining $20 \%$ are tubular which communicate directly with bowel lumen [5].

Several theories have been postulated regarding their origin. As part of the split notochord, an endodermal-ectodermal adhesion between the cord has been proposed to result in persistence of an endomesenchymal tract between yolk sac and the amnion. Some duplications of foregut and hind gut may result from partial twinning. Often duplications, especially that of the ileum, may occur as a result of persistent embryologic diverticuli. Finally intrauterine environmental factors such as trauma, hypoxia during a vascular accident may cause duplications at any level of the gastrointestinal tract [6].

Clinical presentation depend upon the location, type, size and mucosal lining of the duplication. Most of the duplications are diagnosed incidentally while other cases present with a combination of pain and obstructive symptoms [2]. Small cystic duplications can be anchor points for intussusception or can result in volvulus, whereas long tubular duplications with proximal communication drain poorly and retention of intestinal contents can obstruct adjacent intestine. Distal communication is more common

Ultrasonography is an important tool widely used in the evaluation of abdominal pain in children. Duplication cysts are seen as hypoechoic outer muscular layer with an echogenic internal mucosal layer [2]. The diagnosis is often not established before surgery.

The treatment of choice is complete surgical excision with anastomosis [2]. The resection of adjacent bowel is often recommended due to potential complications like ulceration, hemorrhage and malignant changes [7]. Histopathological examination is crucial for establishing the diagnosis and rule out other close differential diagnoses.

\section{CONCLUSION}

Ileal duplication cysts rare anomalies which are often underreported because of their vague and overlapping clinical symptomatology. A high index of clinical suspicion is always needed. Radiological features often are not diagnostic hence histopathological examination forms the corner stone for its identification. This case report highlights an acute presentation of an ileal duplication cyst in a 2 month old baby and hope to contribute to the English literature regarding this entity. 


\section{REFERENCES}

1. Kim, Y. S., Kim, D. J., Bang, S. U., \& Park, J. J. (2016). Intestinal duplication cyst misdiagnosed as meckel's diverticulum. Chinese medical journal, 129(2), 235-6.

2. Basak, A., Ghosh, A., \& Mishra, P. K. (2020). Ileal duplication cyst: a rare cause of intestinal obstruction in infants. Int $J$ Contemp Pediatr. 7:713-715.

3. Basany, L., Aepala, R., Bellary, M. M. R., \& Chitneni, M. (2015). Intestinal obstruction due to ileal duplication cyst and malrotation in a preterm neonate. Journal of Neonatal Surgery, 4(4):48-49.

4. İpek, H., Doğan, G., Yılmaz, D., Metin, M., \& Afşarlar, Ç. E. (2017). A case of asymptomatic ileal duplication cyst associated with acute appendicitis. Journal of pediatric surgery case reports, 22, 25-27.
5. Lee, N. K., Kim, S., Jeon, T. Y., Kim, H. S., Kim, D. H., Seo, H. I., ... \& Jang, H. J. (2010). Complications of congenital and developmental abnormalities of the gastrointestinal tract in adolescents and adults: evaluation with multimodality imaging. Radiographics, 30(6), 1489-1507.

6. Tamvakopoulos, G. S., Sams, V., Preston, P., \& Stebbings, W. S. (2004). Iron-deficiency anaemia caused by an enterolith-filled jejunal duplication cyst. Annals of the Royal College of Surgeons of England, 86(6), W49-51.

7. Shah, N., Lizardo-Escaño, T., Shaaban, H., Dhadham, G., Karki, A., \& Spira, R. (2015). Enterogenous cyst of the small bowel causing intussusception in an adult: Case report and review of literature. Journal of natural science, biology, and medicine, 6(1), 208-210. 